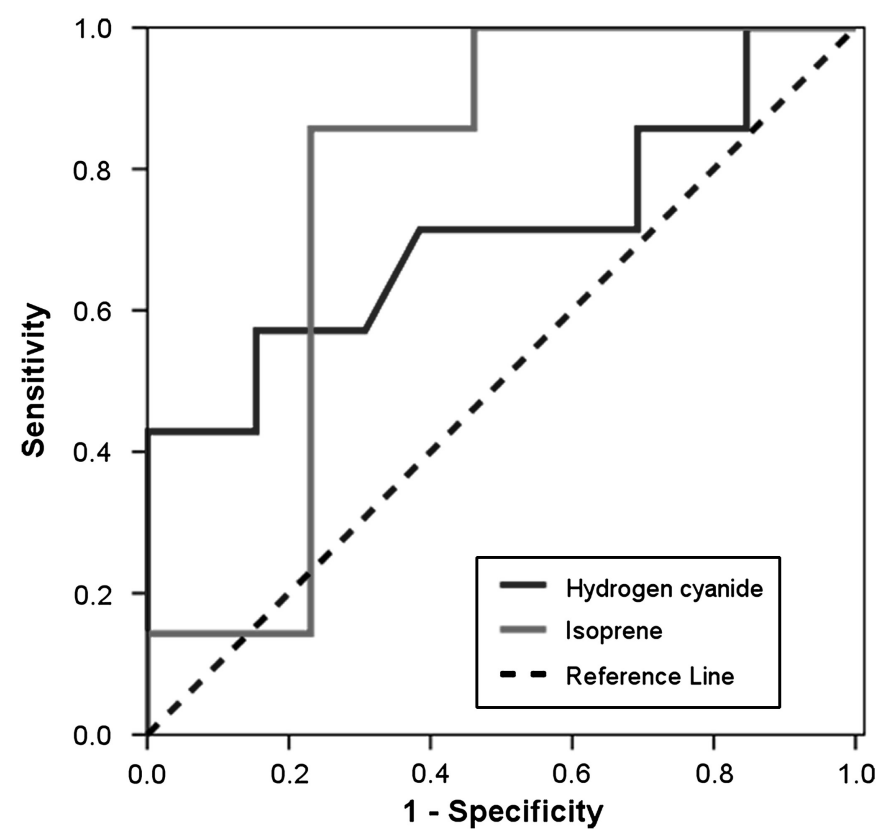

Abstract S128 Figure 1 Receiver operating characteristic (ROC) analysis for hydrogen cyanide and isoprene. Analysis of hydrogen cyanide and isoprene was performed in the exhaled breath of 21 patients with suspected community acquired pneumonia (CAP). A diagnosis of pneumonia was confirmed in 14 patients who met pre-defined criteria for this condition. For patients with suspected CAP and breath hydrogen cyanide $<6.2 \mathrm{ppb}$ sensitivity and specificity were 0.71 and 0.62 respectively (area under ROC curve, 0.71 ). For patients with suspected CAP and breath isoprene $<89 \mathrm{ppb}$ sensitivity and specificity were 0.86 and 0.77 respectively (area under ROC curve, 0.77 ).

Conclusions Results provide evidence that the levels of several prominent breath metabolites, which are potentially related to bacterial activity and lung injury, are significantly altered in patients with this disease. Breath analysis may therefore provide a novel, non-invasive and reliable strategy for the diagnosis of pneumonia.

\section{S129 PREDICTING OUTCOME FROM HIV-ASSOCIATED PNEUMOCYSTIS PNEUMONIA}

doi:10.1136/thx.2010.150946.30

${ }^{1} \mathrm{D}$ Armstrong-James, ${ }^{2} \mathrm{~A} J$ Copas, ${ }^{3} \mathrm{P} \mathrm{D}$ Walzer, ${ }^{4} \mathrm{~S}$ G Edwards, ${ }^{2} \mathrm{R}$ F Miller. ${ }^{1}$ Hospital for Tropical Diseases, London, UK; ${ }^{2}$ University College London, London, UK; ${ }^{3}$ University of Cincinnati, Cincinnati, USA; ${ }^{4}$ Camden Provider Services PCT, London, UK

Background The presentation of Pneumocystis jirovecii pneumonia (PCP) ranges from mild to severe. The former responds to antimicrobial therapy, the latter has a high mortality rate despite treatment. Several studies have described clinical and laboratory factors that are predictive of death from PCP. Our objectives were to create a prognostic scoring model to aid the clinician in predicting outcome from HIV-associated PCP.

Methods A prognostic scoring model was built using risk factors identifiable at/soon after hospitalisation, that is, 'by the bedside'which have previously been identified as being associated with mortality from PCP (a repeat episode of PCP, the patient's age, their haemoglobin $(\mathrm{Hb}), \mathrm{PaO}_{2}$ (breathing room air), both on admission, the presence of co-morbidity (Comorb), such as lymphoma or pregnancy, and the presence of pulmonary Kaposi sarcoma (PKS) (Walzer PD, et al CID 2008;46:625-33). The model was built from data concerning 592 consecutive episodes of PCP that had occurred among 540 patients presenting to a specialist inpatient HIV treatment centre.
Results The prognostic scoring model was: $[25.5+$ (age in years/10) +2 (if a repeat episode of PCP) +4 (if PKS detected) +4 (if Comorb present $)-\mathrm{PaO}_{2}-\mathrm{Hb}$. The prognostic model produced scores ranging from 0 to 20; median (IOR)=9 (7-11). Testing interactions between risk factors and time showed the model to be applicable across all time periods. Patients were divided into five groups according to prognostic score: $0-3.9=$ group $1,4-7.9=$ group 2 , $8-11.9=$ group $3,12-15.9=$ group 4,16 or greater =group 5 . Abstract S129 Table 1 shows mortality rates among the 540 patients with PCP, grouped according to their prognostic scores.

\section{Abstract S129 Table 1}

\begin{tabular}{ll}
\hline Group & Mortality (\%) \\
\hline 1 & 0 \\
2 & 3 \\
3 & 13 \\
4 & 29 \\
5 & 59 \\
\hline
\end{tabular}

Conclusions While this prognostic scoring model requires further validation in patient cohorts from other healthcare institutions, it is potentially a simple 'by the bedside' method of identifying patients early in their hospital admission who are at high and low risk of in-hospital death from PCP and so may aid the clinician in assessing the severity of illness and in deciding on treatment strategies.

\section{S130 OUTCOMES OF PSEUDOMONAS ERADICATION THERAPY IN PATIENTS WITH NON-CYSTIC FIBROSIS BRONCHIECTASIS}

doi:10.1136/thx.2010.150946.31

L White, G Mirrani, M Grover, J Rollason, A Malin, J Suntharalingam. Royal United Hospital, Bath, UK

Introduction and objectives In patients with non-cystic fibrosis (CF) bronchiectasis, Pseudomonas aeruginosa infection is associated with increased hospital admissions, poorer quality of life and accelerated lung function decline. The BTS guidelines recommend early Pseudomonas eradication therapy. However, despite clear evidence in CF, there is little evidence in non-CF bronchiectasis. Since 2004, we have initiated eradication therapy following initial Pseudomonas infection in non-CF bronchiectasis patients. This retrospective study assessed clinical and microbiological outcomes.

Methods All patients undergoing Pseudomonas eradication therapy from 2004 to 2010 were identified from our electronic database. Therapy included intravenous, oral and nebulised antibiotics. Patients were assessed for microbiological eradication, exacerbation frequency (measured by number of antibiotic courses), hospital admissions, clinical symptoms and lung function.

Results 30 patients were identified. $21(67.7 \%)$ were females and mean age was 62.2 years. Median follow-up time was 26.4 months. Eradication therapy involved 2 weeks of intravenous antibiotics (12 patients), intravenous antibiotics followed by oral ciprofloxacin (13 patients) or oral ciprofloxacin alone (five patients), combined with 1-4 months of nebulised colomycin in 26 patients. Pseudomonas was initially eradicated from sputum in 24 patients (80.0\%). 13 patients (43.3\%) remained Pseudomonasfree at latest follow-up. 11 patients (36.7\%) were subsequently reinfected with median time to reinfection of 6.2 months (Abstract S130 Figure 1). 17 patients with chronic infection were maintained on regular nebulised antibiotics. Exacerbation frequency was significantly reduced, with mean number of antibiotic courses per year 3.93 pre-eradication, and 2.09 post- 


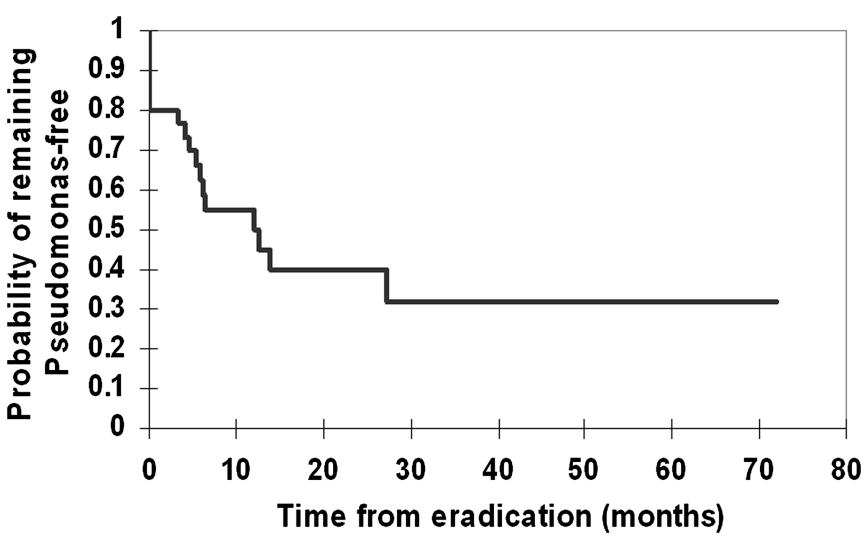

Abstract S130 Figure 1 Kaplan-Meier curve showing Pseudomonasfree time following eradication therapy.

eradication $(p=0.002)$. Mean number of hospital admissions per year were similar, at 0.39 pre-eradication and 0.29 post-eradication $(\mathrm{p}=\mathrm{NS})$. At first follow-up, 20 patients $(66.7 \%)$ reported overall clinical improvement, with reduced cough in 12 (40.0\%), reduced sputum volume in $14(46.7 \%)$ and reduced sputum purulence in $11(36.7 \%)$. Of the 21 patients followed up to 1 year, $6(28.6 \%)$ had further overall improvement and 13 (61.9\%) remained stable. Lung function was not affected by eradication therapy, with mean percentage predicted $\mathrm{FEV}_{1} 62.1 \%$ pre and $64.1 \%$ posteradication $(\mathrm{p}=\mathrm{NS})$.

Conclusion This study demonstrated that Pseudomonas eradication therapy can lead to prolonged clearance of this organism and highly significantly reduces exacerbation rate. This important outcome requires confirmation in a prospective study.

\section{S131 FATIGUE IN BRONCHIECTASIS: ITS RELATIONSHIP TO PSEUDOMONAS COLONISATION, DYSPNOEA AND AIRFLOW OBSTRUCTION}

doi:10.1136/thx.2010.150946.32

${ }^{1} \mathrm{~J} \mathrm{G}$ Macfarlane, ${ }^{1} \mathrm{H}$ Tedd, ${ }^{2} \mathrm{P}$ McAlinden, ${ }^{2} \mathrm{~T}$ Small, ${ }^{2} \mathrm{~L}$ Rostron, ${ }^{3} \mathrm{~J}$ Newton, ${ }^{4} \mathrm{~A}$ De Soyza. ${ }^{1}$ Freeman Hospital Respiratory Department, Newcastle upon Tyne, UK; ${ }^{2}$ William Leech Clinical Research Centre, Newcastle upon Tyne, UK; ${ }^{3}$ Institute of Ageing and Health, Newcastle University, Newcastle upon Tyne, UK; ${ }^{4}$ Institute of Cellular Medicine, Newcastle University, Newcastle upon Tyne, UK

Introduction and objectives Fatigue is a complex and disabling symptom in non-CF bronchiectasis (nCF-Br) and can be formally measured using the Fatigue Impact Scale (FIS). FIS scores of $>40$ out of 120 are clinically significant. The FIS score has been shown to be prognostic of premature death in Primary Biliary cirrhosis but is not linked to markers of organ dysfunction (liver function tests). As poorer outcomes have been recently reported in Pseudomonas infected $\mathrm{nCF}-\mathrm{Br}$ patients we aimed to measure the correlation between FIS scores and parameters of severity in nCF-Br, for example, Pseudomonas infection, degree of dyspnoea and airflow obstruction.

Methods $\mathrm{FEV}_{1} \%$ predicted, MMRC dyspnoea score (MMRCD) and FIS were recorded in stable adult nCF-Br patients attending specialist clinic. All previous sputum cultures isolating Pseudomonas aeruginosa were reviewed. Two groups of patients were studied: those with Pseudomonas 'colonisation' (organism cultured $\geq 2$ occasions, 3 months apart within 1-year period) and those with 'isolation' (organism cultured $\geq 1$ occasion). Statistical comparison used $\chi^{2}$, Fisher's correlation and Mann-Whitney $U$ tests.

Results 73F, 41M patients were included; average age 60 (range 24-90) with an average $\mathrm{FEV}_{1} 66 \%$ predicted (SD +/-26\%). 54
(47\%) patients had Pseudomonas isolation; 38 (33\%) patients had colonisation. Fatigue levels were similar in patients with and without colonisation (median 48.5 vs $36.5, p=0.31$ ). Significant fatigue (FIS $>40$ ) was more common in patients with Pseudomonas isolation (47\%) than those with no previous isolates ( $p=0.04$, $\mathrm{OR}=2.2$ ) However, fatigue levels, although increased, were not significantly different (median FIS 50 vs $32 ; p=0.064$ ). Fatigue correlated with MMRCD $(\mathrm{r}=0.54, \mathrm{p}<0.0001)$ but less well with $\mathrm{FEV}_{1} \%$ predicted $(\mathrm{r}=0.2, \mathrm{p}=0.04) . \mathrm{FEV}_{1} \%$ predicted was lower in patients with Pseudomonas colonisation (median $\mathrm{FEV}_{1} 49 \%$ vs $74 \%$, $\mathrm{p}=0.0007$ ) and in patients with Pseudomonas isolation (median $\mathrm{FEV}_{1}$ $52 \%$ vs $74 \%, p=0.002$ ).

Conclusions Pseudomonas infection (past or present) appears to be associated with greater clinically significant fatigue scores and poorer lung function. Fatigue doesn't strongly correlate with $\mathrm{FEV}_{1} \%$ predicted but is correlated with MMRCD. Further regression analysis of variables is underway to understand these inter relationships further. Systemic aspects of Pseudomonas infection may be different to other infections explaining the divergence.

\section{Clinical and translational observations in asthma}

\section{S132 AIRWAY DYSFUNCTION AND INFLAMMATION IN POOL AND NON-POOL BASED ELITE ENDURANCE ATHLETES}

doi:10.1136/thx.2010.150946.33

${ }^{1} \mathrm{~N}$ Martin, ${ }^{2} \mathrm{M}$ R Lindley, ${ }^{1} \mathrm{~B}$ Hargadon, ${ }^{1} \mathrm{~W}$ Monteiro, ${ }^{1} \mathrm{I} \mathrm{D}$ Pavord. ${ }^{1}$ Instiute for Lung Health, Glenfield Hospital, Leicester, UK; ${ }^{2}$ Department Human Sciences, Loughborough University, Loughborough, UK

Introduction Previous studies have suggested that eosinophilic airway inflammation is common in elite swimmers; the chemical pool environment often blamed. We set out to address this question in a cross-sectional study of 109 international athletes from a variety of sporting backgrounds.

Methods All had symptoms suggesting exercise-induced asthma and were either inhaled corticosteroid naïve or withdrew these for $>4$ weeks. $\beta_{2}$-agonists, exercise and caffeine were withheld for $8 \mathrm{~h}$ prior to testing. Symptoms were assessed using the Juniper ACQ, airways dysfunction using the eucapnic voluntary hyperventilation $(\mathrm{EVH})$ test and airways inflammation using exhaled nitric oxide $\left(\mathrm{FE}_{\mathrm{NO}}\right)$ and induced sputum eosinophil \% (eos). Athletes were classed as pool based if they exercised in an indoor pool environment for $>5 \mathrm{~h}$ per week, and non-pool based if they exercised in a pool for $<1 / 2$ h per week.

Results Demographic details were similar. Mean ( \pm SEM) \% fall in $\mathrm{FEV}_{1}$ post $\mathrm{EVH}$ was $18.96 \pm 1.701(\mathrm{n}=47)$ in pool and $11.39 \pm 1.249$ $(\mathrm{n}=62)$ in non-pool athletes (mean difference $7.569 ; 95 \%$ CI 3.480 to $11.66 ; p=0.0004), 76 \%$ of pool and $39 \%$ of non-pool athletes had a positive test ( $>10 \%$ fall). The geometric mean (log SD) eos (pool $2.667(0.797)$ ), non-pool 3.060 (0.867), $\mathrm{p}=0.802)$, and $\mathrm{FE}_{\mathrm{NO}}$ (pool 25.05 (1.570), non-pool 28.06 (1.475) ppb, p=0.914) was no different between groups; $14.9 \%$ of pool and $12.9 \%$ of non-pool athletes had eos $>3 \%$. \% fall in FEV1 had good correlation with $\log$ eos $(\mathrm{r}=0.551, \mathrm{p}<0.0001)$; a $25 \%$ fall being the optimum (AUC $0.89, \mathrm{p}<0.0001$, sens $79 \%$, spec 93\%). Log $\mathrm{FE}_{\mathrm{NO}}$ and $\log$ eos correlated strongly $(\mathrm{r}=0.644, \mathrm{p}<0.0001) ; \mathrm{FE}_{\mathrm{NO}}$ of $>47$ being most predictive of eos $>3 \%$ (AUC $0.912, p<0.0001$, sens $78 \%$, spec $92 \%$ ). Symptoms correlated poorly with either airways dysfunction or inflammation.

Conclusions Individual athletes with symptoms vary markedly in the levels of airways dysfunction and inflammation expressed. There is more airway dysfunction in pool athletes but not more eosinophilic airways inflammation suggesting that the pool 